\title{
THE CONVERGENCE OF AN IMPLICIT MEAN VALUE ITERATION
}

B. E. RHOADES AND ŞTEFAN M. ŞOLTUZ

Received 3 April 2006; Revised 10 May 2006; Accepted 11 May 2006

We show the convergence of an implicit mean value iteration when applied to uniformly pseudocontractive maps. Remarks about other implicit mean value iterations are given.

Copyright @ 2006 Hindawi Publishing Corporation. All rights reserved.

\section{Introduction}

Let $X$ be a real Banach space, $T: X \rightarrow X$ a map, and $x_{0}, u_{0} \in X$. In [5], the following iteration is introduced:

$$
u_{n+1}=\left(1-\alpha_{n}\right) u_{n}+\alpha_{n} T u_{n}
$$

where $\left\{\alpha_{n}\right\} \subset(0,1)$.

In the rest of the paper, we will assume that $(I-t T)^{-1}$ exists for all $t \in(0,1)$. Consider the following iteration, see [8]:

$$
x_{n+1}=\left(1-\alpha_{n}\right) x_{n}+\alpha_{n} T x_{n+1},
$$

where the sequence $\left\{\alpha_{n}\right\}$ is in $(0,1)$.

The following example shows that iteration (1.2) is well defined. We recall the following well-known result.

Lemma 1.1. Let $\left\{\beta_{n}\right\}$ be a nonnegative sequence such that $\beta_{n} \in(0,1]$, for all $n \in \mathbb{N}$. If $\sum_{n=1}^{\infty} \beta_{n}=\infty$, then $\prod_{n=1}^{\infty}\left(1-\beta_{n}\right)=0$.

Inspired by $[3,8]$, we give an example which shows that Mann iterations (1.1) and (1.2) are independent.

Example 1.2. Let $X=\mathbb{R}^{2}$. Let $T: X \rightarrow X$ be the map given by

$$
T(x, y)=\left(\begin{array}{cc}
1 & 1 \\
-1 & 1
\end{array}\right)\left(\begin{array}{l}
x \\
y
\end{array}\right), \quad \forall(x, y) \in \mathbb{R}^{2} .
$$


2 The convergence of an implicit mean value iteration

Iteration (1.1) is not convergent to the fixed point of $T$, while iteration (1.2), for $\left\{\alpha_{n}\right\} \subset$ $(1 / 2,1)$, converges to the fixed point of $T$.

Proof. Let $u=(x, y)$. For all $\lambda \in] 0,1[$,

$$
\begin{aligned}
|(1-\lambda) u+\lambda T u|^{2} & =|(1-\lambda)(x, y)+\lambda T(x, y)|^{2} \\
& =|(1-\lambda)(x, y)+\lambda(x+y,-x+y)|^{2} \\
& =|(1-\lambda) x+\lambda x+\lambda y,(1-\lambda) y-\lambda x+\lambda y|^{2} \\
& =|(x+\lambda y, y-\lambda x)|^{2}=\left(1+\lambda^{2}\right) x^{2}+\left(1+\lambda^{2}\right) y^{2} \\
& =\left(1+\lambda^{2}\right)|u|^{2}>|u|^{2} .
\end{aligned}
$$

Hence the Mann iteration is not convergent to $(0,0)$, for all $\left\{\alpha_{n}\right\} \subset(0,1)$. Note that $\left(I_{2}-t T\right)^{-1}$ exists for all $t \in(0,1)$. Moreover,

$$
\begin{gathered}
\left(I_{2}-t T\right)=\left(\begin{array}{cc}
1-t & -t \\
t & 1-t
\end{array}\right), \\
\left(I_{2}-t T\right)^{-1}=\frac{1}{(1-t)^{2}+t^{2}}\left(\begin{array}{cc}
1-t & t \\
-t & 1-t
\end{array}\right) .
\end{gathered}
$$

Thus, for $\left\{\alpha_{n}\right\} \in(0,1)$,

$$
x_{n+1}=\frac{1-\alpha_{n}}{\left(1-\alpha_{n}\right)^{2}+\alpha_{n}^{2}}\left(\begin{array}{cc}
1-\alpha_{n} & \alpha_{n} \\
-\alpha_{n} & 1-\alpha_{n}
\end{array}\right) x_{n} .
$$

Denote $x_{n}:=\left(a_{n}, b_{n}\right)^{\prime}$ to obtain

$$
\begin{aligned}
\left|x_{n+1}\right|^{2} & =a_{n+1}^{2}+b_{n+1}^{2} \\
& =\frac{\left(1-\alpha_{n}\right)^{2}}{\left[\left(1-\alpha_{n}\right)^{2}+\alpha_{n}^{2}\right]^{2}}\left[\left(\left(1-\alpha_{n}\right) a_{n}+\alpha_{n} b_{n}\right)^{2}+\left(-\alpha_{n} a_{n}+\left(1-\alpha_{n}\right) b_{n}\right)^{2}\right] \\
& =\frac{\left(1-\alpha_{n}\right)^{2}}{\left[\left(1-\alpha_{n}\right)^{2}+\alpha_{n}^{2}\right]^{2}}\left[\left(1-\alpha_{n}\right)^{2}+\alpha_{n}^{2}\right]\left(a_{n}^{2}+b_{n}^{2}\right)=\frac{\left(1-\alpha_{n}\right)^{2}}{\left[\left(1-\alpha_{n}\right)^{2}+\alpha_{n}^{2}\right]}\left(a_{n}^{2}+b_{n}^{2}\right) \\
& =\left(1-\frac{\alpha_{n}^{2}}{\left(1-\alpha_{n}\right)^{2}+\alpha_{n}^{2}}\right)\left|x_{n}\right|^{2} \leq\left(1-\alpha_{n}\right)\left|x_{n}\right|^{2} .
\end{aligned}
$$

The last inequality holds because $0 \geq 2 \alpha_{n}^{2}-3 \alpha_{n}+1$ for $\alpha_{n} \in(1 / 2,1)$. Lemma 1.1 assures that $\lim _{n \rightarrow \infty}\left|x_{n}\right|=0$. 
Take $T:[0,1) \rightarrow[0,1), T x=x^{2}$, to obtain a map for which Mann iteration converges to the fixed point, while implicit Mann iteration is not well defined and consequently does not converge at all. Using (1.2),

$$
x_{n+1}=\left(1-\alpha_{n}\right) x_{n}+\alpha_{n} x_{n+1}^{2}
$$

Solving for $x_{n+1}$ yields

$$
x_{n+1}=\frac{1-\left(1-4 \alpha_{n}\left(1-\alpha_{n}\right) x_{n}\right)^{1 / 2}}{2 \alpha_{n}}
$$

or

$$
x_{n+1}=\frac{1+\left(1-4 \alpha_{n}\left(1-\alpha_{n}\right) x_{n}\right)^{1 / 2}}{2 \alpha_{n}} .
$$

In the later case, $x_{n+1}$ is no longer inside the interval $[0,1)$. Suppose one always takes the first case. With the choice that each $\alpha_{n}=1 / 2$, we have

$$
x_{n+1}=1-\left(1-x_{n}\right)^{1 / 2}
$$

that is, $1-x_{n+1}=\left(1-x_{n}\right)^{1 / 2}$. Set $a_{n}=1-x_{n}>0$ to obtain $\lim _{n \rightarrow \infty} a_{n}=0$. Thus, $\left\{x_{n}\right\}$ converges to 1 , which is not in the interval $[0,1)$.

The map $J: X \rightarrow 2^{X^{*}}$, given by $J(x):=\left\{f \in X^{*}:\langle x, f\rangle=\|x\|^{2},\|f\|=\|x\|\right\}$, for all $x \in X$, is called the normalized duality mapping. It is easy to see that

$$
\langle y, j(x)\rangle \leq\|x\|\|y\|, \quad \forall x, y \in X, \forall j(x) \in J(x) .
$$

Define

$$
\Psi:=\{\psi \mid \psi:[0,+\infty) \longrightarrow[0,+\infty) \text { is a strictly increasing map such that } \psi(0)=0\}
$$

The following definition can be found, for example, in [4].

Definition 1.3. A map $T: X \rightarrow X$ is called uniformly pseudocontractive if there exist a map $\psi \in \Psi$ and a $j(x-y) \in J(x-y)$ such that

$$
\langle T x-T y, j(x-y)\rangle \leq\|x-y\|^{2}-\psi(\|x-y\|), \quad \forall x, y \in X .
$$

Taking $\psi(a):=\psi(a) \cdot a$, for all $a \in[0,+\infty),(\psi \in \Psi)$, gives the usual definitions of $\psi$-strongly pseudocontractivity. The choice $\psi(a):=\gamma \cdot a^{2}, \gamma \in(0,1)$, for all $a \in[0,+\infty)$, $(\psi \in \Psi)$, yields the usual definition of strong pseudocontractivity.

The convergence of (1.2) dealing with strongly pseudocontractive maps was proved in [8]. We will prove the convergence of iteration (1.2) when applied to uniformly pseudocontractive maps. For this purpose, we need the following result. 
4 The convergence of an implicit mean value iteration

Lемма 1.4 [9]. Let $\left\{a_{n}\right\}$ be a nonnegative bounded sequence which satisfies the following inequality:

$$
a_{n+1} \leq\left(1-\alpha_{n}\right) a_{n}+\alpha_{n} a_{n+1}-\alpha_{n} \frac{\psi\left(a_{n+1}\right)}{a_{n+1}}+\alpha_{n} \varepsilon_{n}, \quad \forall n \geq n_{0},
$$

where $\psi(\cdot) \in \Psi, \alpha_{n} \in(0,1), \varepsilon_{n} \geq 0$, for all $n \in \mathbb{N}, \sum_{n=0}^{\infty} \alpha_{n}=\infty$, and $\lim _{n \rightarrow \infty} \varepsilon_{n}=0$. Then $\lim _{n \rightarrow \infty} a_{n}=0$.

\section{Main results}

Theorem 2.1. Let $X$ be a real Banach space and $T: X \rightarrow X$ a uniformly pseudocontractive map with a fixed point such that

$$
\exists(I-t T)^{-1} \quad \forall t \in(0,1) .
$$

If $\left\{\alpha_{n}\right\} \subset(0,1)$ satisfies

$$
\lim _{n \rightarrow \infty} \alpha_{n}=0, \quad \sum_{n=0}^{\infty} \alpha_{n}=\infty,
$$

and $\left\{x_{n}\right\}$ is bounded, then for $x_{0} \in X$ the iteration (1.2) converges to the fixed point of $T$.

Proof. The uniqueness of the fixed point comes from (1.14). Let $x^{*}$ be the fixed point of $T$.

If there exists a nonnegative integer $n$ for which $x_{n}=x^{*}$, then from (1.2),

$$
x_{n+1}=\left(1-\alpha_{n}\right) x^{*}+\alpha_{n} T x_{n+1}
$$

or

$$
\left(I-\alpha_{n} T\right) x_{n+1}=\left(I-\alpha_{n} T\right) x^{*}
$$

which, using (2.1), implies that $x_{n+1}=x^{*}$. By induction, $x_{m}=x^{*}$ for all $m \geq n$.

We may therefore assume that each $x_{n} \neq x^{*}$. Using (1.2)-(1.14),

$$
\begin{aligned}
\left\|x_{n+1}-x^{*}\right\|^{2} & =\left\langle x_{n+1}-x^{*}, j\left(x_{n+1}-x^{*}\right)\right\rangle \\
& =\left\langle\left(1-\alpha_{n}\right)\left(x_{n}-x^{*}\right)+\alpha_{n}\left(T x_{n+1}-T x^{*}\right), j\left(x_{n+1}-x^{*}\right)\right\rangle \\
& =\left(1-\alpha_{n}\right)\left\langle\left(x_{n}-x^{*}\right), j\left(x_{n+1}-x^{*}\right)\right\rangle+\alpha_{n}\left\langle T x_{n+1}-T x^{*}, j\left(x_{n+1}-x^{*}\right)\right\rangle \\
& \leq\left(1-\alpha_{n}\right)\left\|x_{n}-x^{*}\right\|\left\|x_{n+1}-x^{*}\right\|+\alpha_{n}\left\|x_{n+1}-x^{*}\right\|^{2}-\alpha_{n} \psi\left(\left\|x_{n+1}-x^{*}\right\|\right) \\
& \leq\left\|x_{n+1}-x^{*}\right\|\left(\left(1-\alpha_{n}\right)\left\|x_{n}-x^{*}\right\|+\alpha_{n}\left\|x_{n+1}-x^{*}\right\|-\alpha_{n} \frac{\psi\left(\left\|x_{n+1}-x^{*}\right\|\right)}{\left\|x_{n+1}-x^{*}\right\|}\right) .
\end{aligned}
$$

Dividing by $\left\|x_{n+1}-x^{*}\right\|$ and defining $a_{n}=\left\|x_{n}-x^{*}\right\|$ yield (1.15) with each $\varepsilon_{n}=0$. From Lemma $1.4, \lim _{n \rightarrow \infty} a_{n}=0$. 
The following remark indicates some ways in which Theorem 2.1 can be applied to certain accretive maps.

Remark 2.2. (1) The operator $T$ is a (uniformly, $\psi$-strongly, strongly) pseudocontractive map if and only if $(I-T)$ is a (uniformly, $\psi$-strongly, strongly) accretive map.

(2) Let $T, S: X \rightarrow X$, and $f \in X$ be given. A fixed point for the map $T x=f+(I-S) x$, for all $x \in X$, is a solution for $S x=f$, and conversely.

(3) Consider iteration (1.2) with $T x=f+(I-S) x$ to obtain a convergence result to the solution of $S x=f$.

(4) Let $f \in X$ be given. If the operator $S$ is accretive, then $f-S$ is a strongly pseudocontractive map.

(5) Let $T, S: X \rightarrow X$. A fixed point for the map $T x=f-S x$, for all $x \in X$, is a solution for $x+S x=f$, and conversely.

(6) Consider iteration (1.2) with $T x=f-S x$ to obtain a convergence result to the solution of $x+S x=f$.

Remark 2.3. If (1.14) is also true for all $x \in X$, and $y:=x^{*}$, the fixed point, then such a map is called uniformly hemicontractive. Obviously, our result holds for the uniformly hemicontractive case.

\section{Remarks about implicit mean value iterations}

Let $X$ be a real Banach space and $B$ a nonempty convex subset, $u_{0}, x_{0} \in B$. Consider for $\left\{\alpha_{n}\right\} \subset(0,1)$, a finite family of functions $\left\{T_{i}\right\}_{i=1}^{N}: B \rightarrow B$ and the following two iterations:

$$
\begin{gathered}
x_{1}=\alpha_{1} x_{0}+\left(1-\alpha_{1}\right) T_{1} x_{1}, \\
x_{2}=\alpha_{2} x_{1}+\left(1-\alpha_{2}\right) T_{2} x_{2}, \\
\vdots \\
x_{N}=\alpha_{N} x_{N-1}+\left(1-\alpha_{N}\right) T_{N} x_{N}, \\
x_{N+1}=\alpha_{N+1} x_{N}+\left(1-\alpha_{N+1}\right) T_{1} x_{N+1}, \\
\vdots \\
u_{1}=\alpha_{1} u_{0}+\left(1-\alpha_{1}\right) T_{1} u_{1}, \\
u_{2}=\alpha_{2} u_{1}+\left(1-\alpha_{2}\right) T_{2} u_{2}, \\
\vdots \\
x_{N}=\alpha_{N} x_{N-1}+\left(1-\alpha_{N}\right) T_{N} x_{N}, \\
x_{N+1}=\alpha_{N+1} x_{N}+\left(1-\alpha_{N+1}\right) T_{1}^{2} x_{N+1}, \\
\vdots \\
x_{2 N}=\alpha_{2 N} x_{2 N-1}+\left(1-\alpha_{2 N}\right) T_{N}^{2} x_{2 N},
\end{gathered}
$$


Iteration (3.1) has been considered in $[2,6,7,11,12]$. Iteration (3.1) has been discussed in $[1,10]$. Note that iteration (1.2) is a particular case of (3.1). However, as far as we know, no such paper is dedicated to the convergence of the implicit iteration dealing with uniformly pseudocontractive maps.

Condition (2.1) forces iteration (1.2) to be well defined. The papers listed above do not impose such a condition, and consequently, the resulting implicit mean value iterations need not be well defined, as the following example illustrates.

Example 3.1. Take $T_{i}:[0,1] \rightarrow[0,1], i=1,2, T_{1}(x)=x^{2}$, and $T_{2}(x)=(1 / 2) x$ with the common fixed point $x^{*}=0$. Then for $x_{0}=1, x_{1}=\alpha_{1} x_{0}+\left(1-\alpha_{1}\right) x_{1}^{2}$, one obtains $x_{1}=$ 1 and $x_{1}=\alpha_{1} /\left(1-\alpha_{1}\right)$. Take now $u_{0}=1, u_{1}=\alpha_{1} u_{0}+\left(1-\alpha_{1}\right) u_{1}^{2}$, and $u_{2}=\alpha_{2} u_{1}+(1-$ $\left.\alpha_{2}\right)(1 / 4) u_{2}$. Observe that there are two possible values for $u_{2}$.

Remark 3.2. The existence of $\left(I-t T_{i}\right)^{-1}$, for all $\left.t \in\right] 0,1[, i=1, N$, should be added to the hypotheses of the results of $[2,6,7,11,12]$ in order to have well-defined iterations. The existence of $\left(I-t T_{i}^{p}\right)^{-1}$, for all $t \in(0,1), i=1, N$, for all $p \geq 1$, should be added to the hypotheses of the results of $[1,10]$.

\section{Acknowledgment}

The authors are indebted to the referees for carefully reading the paper and for making useful suggestions.

\section{References}

[1] S. S. Chang, K. K. Tan, H. W. J. Lee, and C. K. Chan, On the convergence of implicit iteration process with error for a finite family of asymptotically nonexpansive mappings, Journal of Mathematical Analysis and Applications 313 (2006), no. 1, 273-283.

[2] R. Chen, Y. Song, and H. Zhou, Convergence theorems for implicit iteration process for a finite family of continuous pseudocontractive mappings, Journal of Mathematical Analysis and Applications 314 (2006), no. 2, 701-709.

[3] C. E. Chidume and S. A. Mutangadura, An example of the Mann iteration method for Lipschitz pseudocontractions, Proceedings of the American Mathematical Society 129 (2001), no. 8, 23592363.

[4] Łj. Ćirić and J. S. Ume, Iterative processes with errors for nonlinear equations, Bulletin of the Australian Mathematical Society 69 (2004), no. 2, 177-189.

[5] W. R. Mann, Mean value methods in iteration, Proceedings of the American Mathematical Society 4 (1953), no. 3, 506-510.

[6] M. O. Osilike, Implicit iteration process for common fixed points of a finite family of pseudocontractive maps, Panamerican Mathematical Journal 14 (2004), no. 3, 89-98.

[7] _ Implicit iteration process for common fixed points of a finite family of strictly pseudocontractive maps, Journal of Mathematical Analysis and Applications 294 (2004), no. 1, 73-81.

[8] Ş. M. Şoltuz, The backward Mann iteration, Octogon Mathematics Magazine 9 (2001), no. 2, 797-800.

[9] _ New technique for proving the equivalence of Mann and Ishikawa iterations, Revue d'Analyse Numérique et de Théorie de l'Approximation 34 (2005), no. 1, 103-108.

[10] Z.-H. Sun, Strong convergence of an implicit iteration process for a finite family of asymptotically quasi-nonexpansive mappings, Journal of Mathematical Analysis and Applications 286 (2003), no. $1,351-358$. 
[11] H.-K. Xu and R. G. Ori, An implicit iteration process for nonexpansive mappings, Numerical Functional Analysis and Optimization 22 (2001), no. 5-6, 767-773.

[12] Y. Zhou and S. S. Chang, Convergence of implicit iteration process for a finite family of asymptotically nonexpansive mappings in Banach spaces, Numerical Functional Analysis and Optimization 23 (2002), no. 7-8, 911-921.

B. E. Rhoades: Department of Mathematics, Indiana University, Bloomington, IN 47405-7106, USA E-mail address: rhoades@indiana.edu

Ştefan M. Şoltuz: "Numerical Analysis and Application Theory Workgroup, Tiberiu Popoviciu Institute of Numerical Analysis," P.O. Box 68-1, 400110 Cluj-Napoca, Romania

E-mail address: smsoltuz@gmail.com 


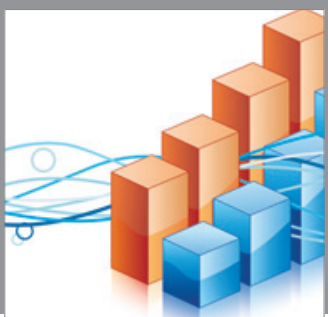

Advances in

Operations Research

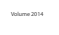

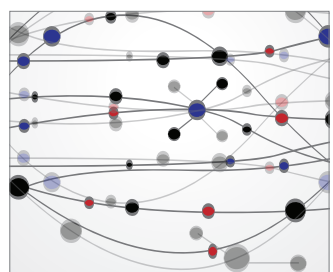

\section{The Scientific} World Journal
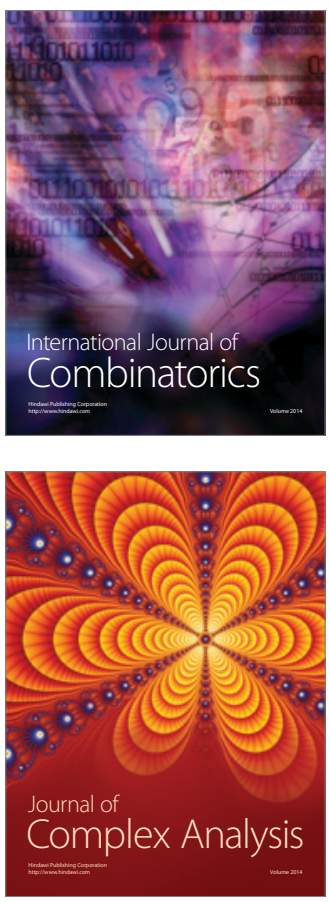

International Journal of

Mathematics and

Mathematical

Sciences
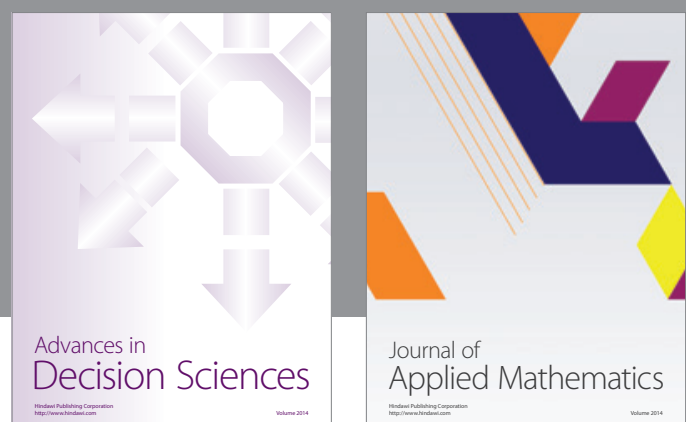

Journal of

Applied Mathematics
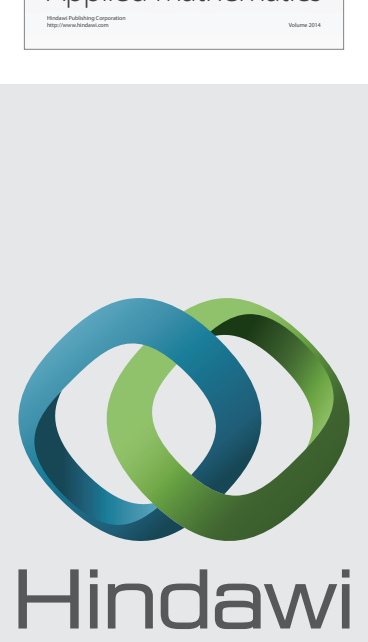

Submit your manuscripts at http://www.hindawi.com
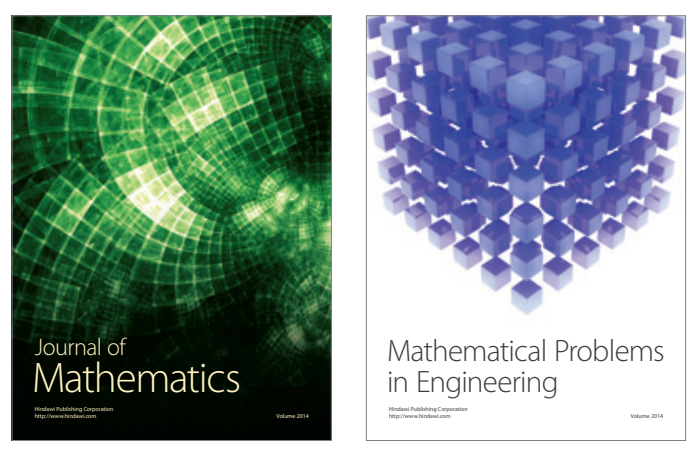

Mathematical Problems in Engineering
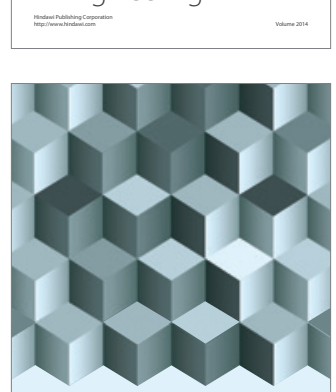

Journal of

Function Spaces
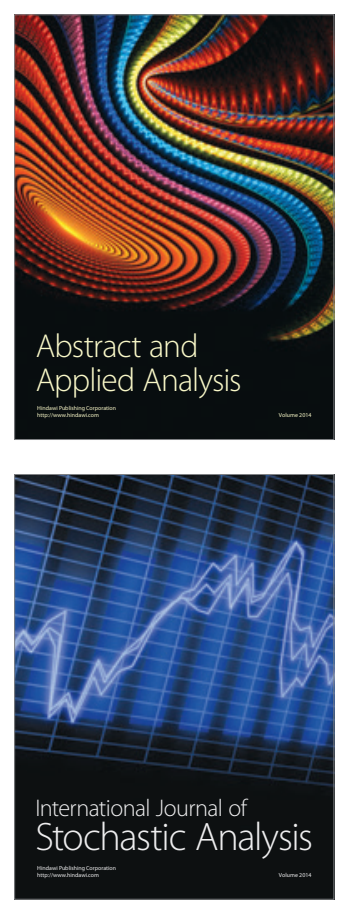

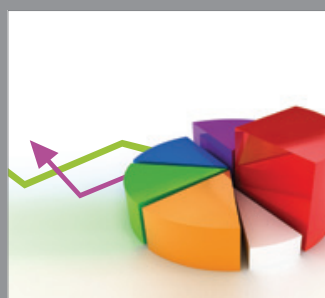

ournal of

Probability and Statistics

Promensencen
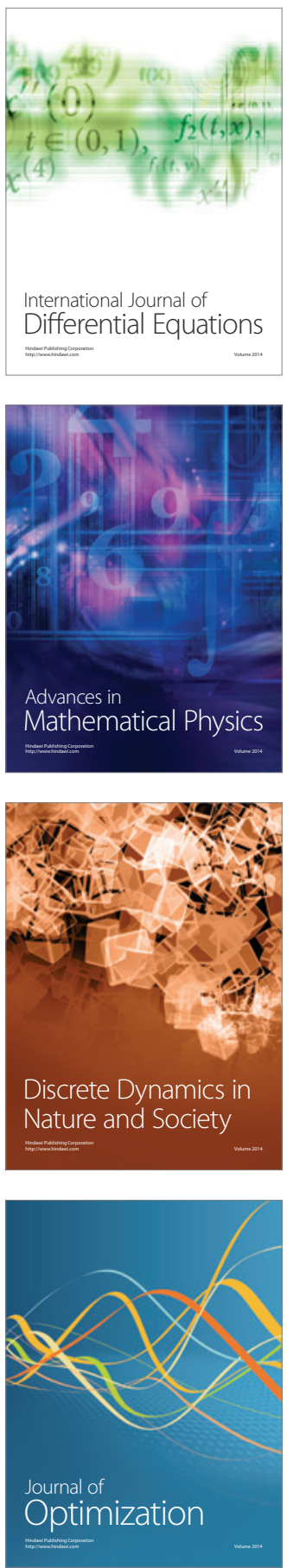